\title{
Trotz Schuldenkrise: Wachstum bei Freizeit und Reisen ungebrochen
}
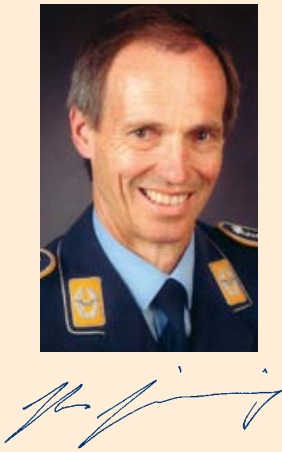

Liebe Kolleginnen, liebe Kollegen,

trotz der Turbulenzen und der Unwägbarkeiten auf den Finanzmärkten, der unsicheren Finanzierung der Staatshaushalte und der Sparzwänge für alle Volkswirtschaften ist die Bereitschaft für Ausgaben bei den privaten Haushalten - vor allem in Deutschland - ungebrochen. Investitionen für Reisen und sportliche Freizeitgestaltung verzeichnen erstaunliche Zuwächse. Demzufolge sind das Engagement und die Betreuungs- und Beratungsleistungen unserer 6 Fachgesellschaften weiterhin und zunehmend sowohl notwendig wie unverzichtbar.

Forschung, Ausbildung, Begutachtung und Betreuung für die Arbeit und Leistung der Dienstleister, welche Millionen Reisende zu Wasser, zu Lande und in der Luft sicher befördern und begleiten, haben an Bedeutung zugenommen. Denn neben dem Anspruch der Nutzer hat auch der Gesetzgeber hohe Qualitätsnormen festgelegt. Dafür haben unsere Fachgesellschaften Arbeitsgemeinschaften und Qualitätszirkel eingerichtet. Außerdem nutzen sie die Fachexpertise universitärer Einrichtungen, von Verbänden und der Industrie. Ziel muss es sein, dass unsere Expertise nicht nur von Individuen, sondern auch von Politik und Wirtschaft genutzt wird - bei Gesetzen, Verordnungen und Produktentwicklung. So werden wir unsere Bedeutung erhalten und stärken.

Dipl.-Ing. Christoph Sevenich informiert im vorliegenden Heft über die Trinkwasserqualität auf Kreuzfahrtschiffen. Das kann beim Zusammenleben von vielen Tausend Menschen auf engstem Raum und dem Bedarf von bis zu 2501 Trinkwasser pro Person und Tag ein großes Problem sein. Sportklettern ist auch für Kinder und Jugendliche eine Boomsportart. Dr. Thomas Hochholzer aus Innsbruck und PD Dr. Volker Schöffl aus Bamberg machen klar, dass Überlastungserscheinungen an Fingergelenken und Fehlhaltungen der Wirbelsäule nicht selten sind und zu zwar zeitlich befristeten, aber durchaus längerem Kletterverbot führen müssen. PD Dr. Tomas Jelinek aus Berlin stellt uns Plasmodium knowlesi, den fünften humanpathogenen Malariaparasiten, vor. Er kann vom Affen auf den Menschen übertragen werden und dann zu schweren Malariaanfällen führen. Eine Behandlung ist mit den gängigen Malariamedikamenten möglich. Ein medizinhistorischer Artikel von Dr. Marion Hulverscheidt aus Berlin über die Forschung für einen Malaria-Impfstoff im Nationalsozialismus mittels Versuchen am Menschen schließt dieses Heft ab.

Ich wünsche Ihnen viel Spaß beim Lesen. Bitte scheuen Sie sich nicht, eigene Artikel einzureichen.

Ihr Dr. Klaus Kimmich, Breisach 